\title{
Matrix Array Transducers in Fetal Heart Imaging
}

\author{
Fabrizio Taddei, Laura Franceschetti, Giuliano Farina, Federico Prefumo, \\ Marino Signorelli, Nicola Fratelli, Caterina Groli
}

\author{
Fetal Cardiology Unit, Department of Obstetrics and Gynecology \\ University of Brescia, Italy
}

Corresponding author: Dr Fabrizio Taddei, Dipartimento di Ostetricia e Ginecologia, Spedali Civili, Piazzale Spedali Civili, 1 25123 Brescia, Italy, Phone +39030 3995365, Fax +39030 3995340

E-mail taddei.fabrizio@tiscali.it

\section{INTRODUCTION}

According to the Oxford English Dictionary, 'real-time' means the actual time during which a process or event takes place, particularly an event or process analyzed by a computer. ${ }^{1}$ As opposed to 'real time', 'subsequent time' indicates a later time at which an event can be elaborated by a computer or at which a recording can be reproduced.

For what specifically concerns ultrasound imaging, the expression 'real-time' should be reserved to describe the ability of an ultrasound system to image moving anatomical structures at the exact time when anatomical data are acquired, or in the worst case to do it with a negligible time delay between acquisition and visualization. This would imply that $2 \mathrm{D}$ images or $3 \mathrm{D}$ volumes are refreshed with a frequency equal or higher to human retinal vision persistence.

\section{Real-time 3D with Matrix Array Probes}

The recent introduction of matrix array probes in fetal ultrasound has made possible a new modality of volume acquisition and

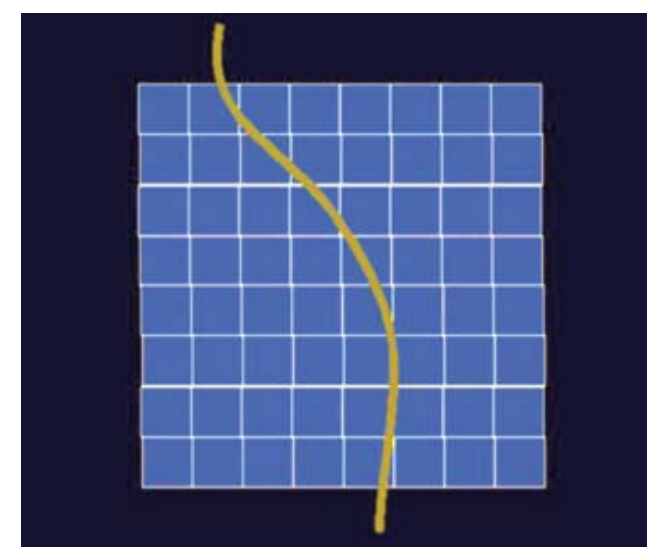

Fig. 1: Frontal view of a matrix array probe. Each single square element represents a piezoelectric crystal. The gray line is the schematic representation of a thin thread lying on the transducer's surface elaboration. In matrix array probes, laser is used to cut the piezoelectric crystal into many equal-sized square elements, forming an element matrix (Fig. 1). These elements are housed in the tip of the transducer so that they can be in close contact with the surface of the body for easy transmission and reception of ultrasound pulses. Each single element can fire an ultrasound beam in all possible directions of space (Figs 2A and B). By appropriately defining the ultrasound beam for each single element, it is possible to build up a pyramidal volumetric ultrasound beam, with an opening angle between $6^{\circ}$ and $100^{\circ}$ (Fig. 3).

This volumetric ultrasound beam allows to obtain a realtime three-dimensional moving image (real-time 3D) without the use of software-reconstructed section planes. Each single plane composing the ultrasound beam is virtually contiguous with the neighboring planes, and there are therefore no empty spaces between each other. This allows voxels to have the same resolution along all of the three directions of the beam (isotropic voxel).

Two orthogonal reference planes are used to localize cardiac structures within the volume. Navigation by cutting within the volume allows to obtain surface rendering views of the intracardiac structures, and to have new 'internal point of view' projections of the atrial and ventricular septa, and of the valves. Moreover, by cutting and rotating the three-dimensional pyramid it is possible to visualize, from a single volume, the ascending aorta and the pulmonary trunk (Figs 4A to F).

\section{Biplane Real-time Imaging}

In this modality, the matrix array transducer allows the simultaneous visualization of two planes, oriented in different directions, with the same degree of resolution (Fig. 5). Images are shown on a screen divided in two parts: the original plane is on the left-hand side, while the right-hand side shows one of the different scanning planes that the sonographer can visualize using a different orientation of the ultrasound beam in the space (axial planes, sagittal planes or rotation). 


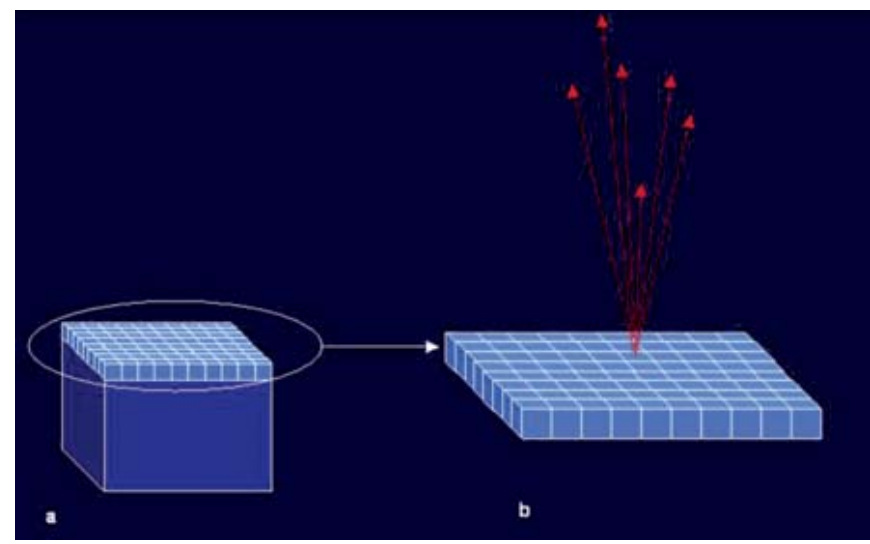

Figs $2 \mathrm{~A}$ and $\mathbf{B}$ : Three-dimensional schematic representation of the matrix array transducer $(A)$ and of the independent emission of ultrasound beams by each single matrix element $(B)$

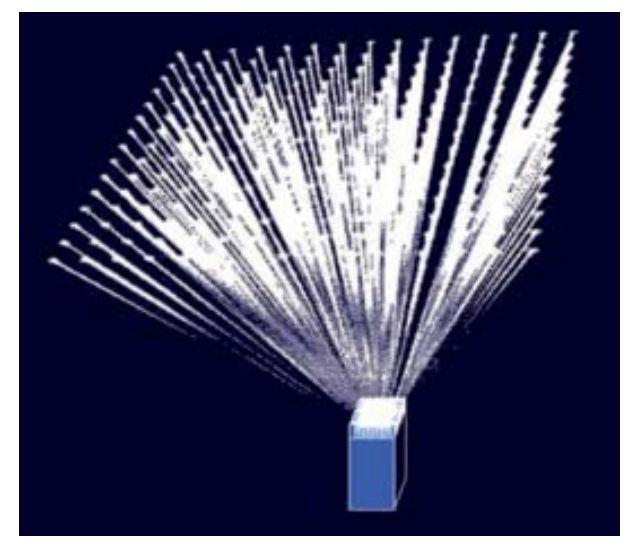

Fig. 3: Pyramidal volumetric ultrasound beam produced by the matrix array transducer, composed by the beams emitted by each single element

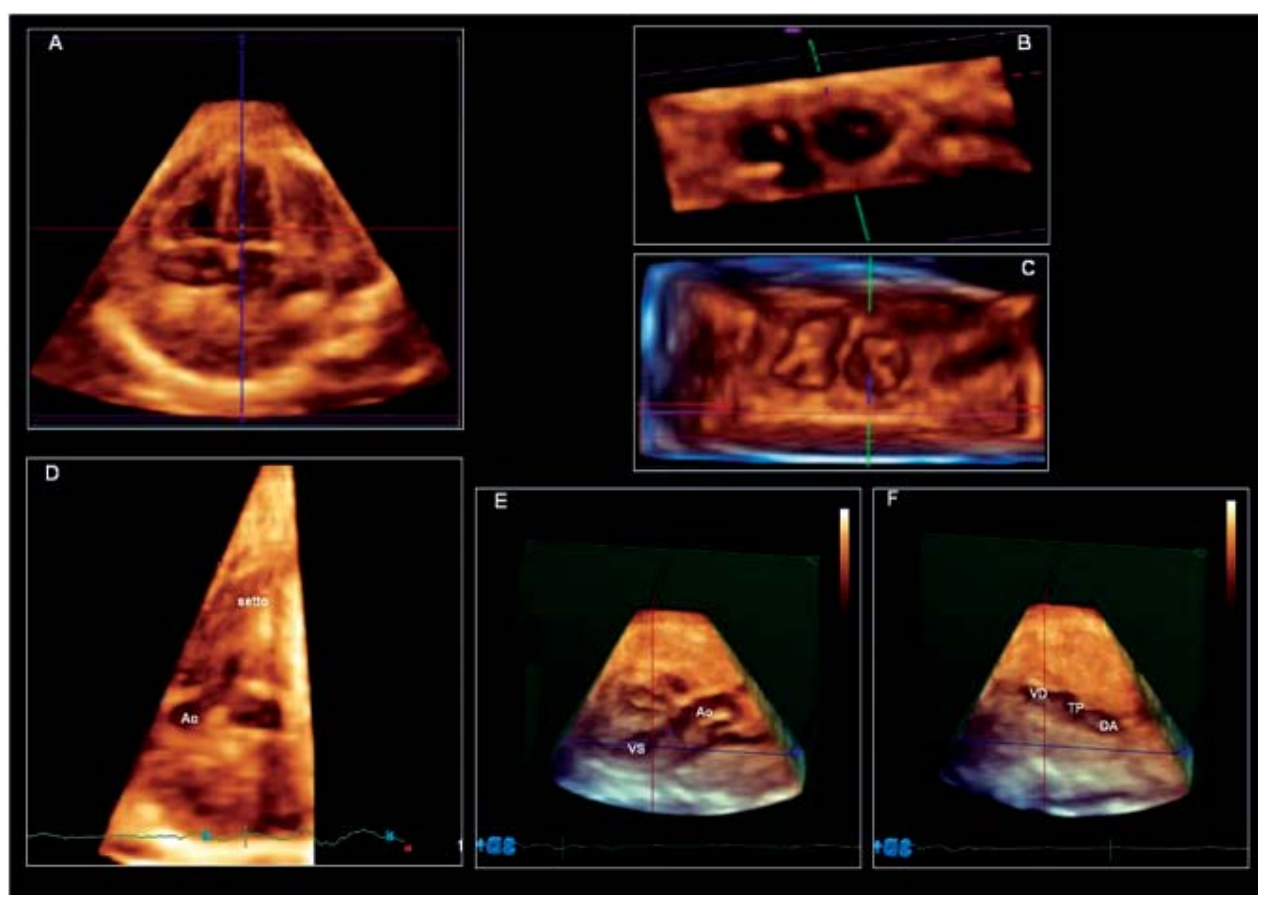

Figs 4A to F: Real time 3D. Demonstration of the four-chamber view (A); atrio-ventrivular valves seen from within the ventricles during diastole $(B)$ and systole $(C)$; interventricular septum with aortic outflow (Ao) (D); left ventricle (VS) and ascending aorta (Ao) (E); right ventricle (VD), pulmonary trunk (TP) and ductus arteriosus (DA) (F)

This second plane can be perpendicular to the main scanning plane, but also parallel or oblique. In this way, starting for example from an axial view of the fetal thorax showing the 4 chambers, and orientating the second plane (simultaneously shown on the right-hand side of the screen) in different directions all the structures of the fetal heart can be visualized (Figs 6 and 7).

\section{CONCLUSION}

The feasibility of real-time 3D ultrasound fetal echocardiography has already been demonstrated in the fetus. ${ }^{2-9}$ The impact of this technology in the clinical management of cardiac abnormalities is far from being defined. At present, the use matrix array transducers is limited by their low working 


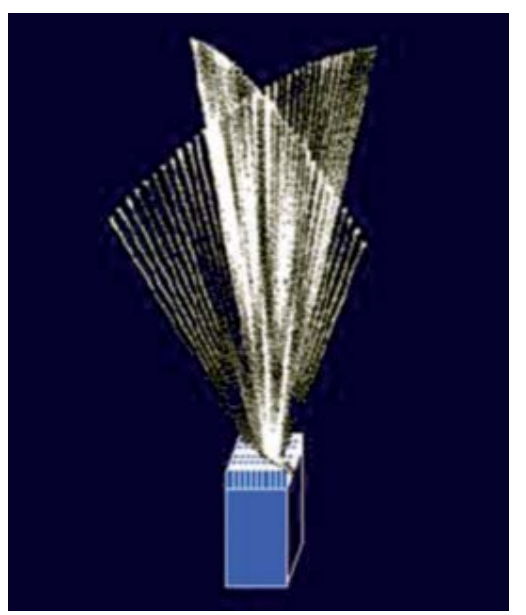

Fig. 5: Schematic representation of the biplane real-time mode

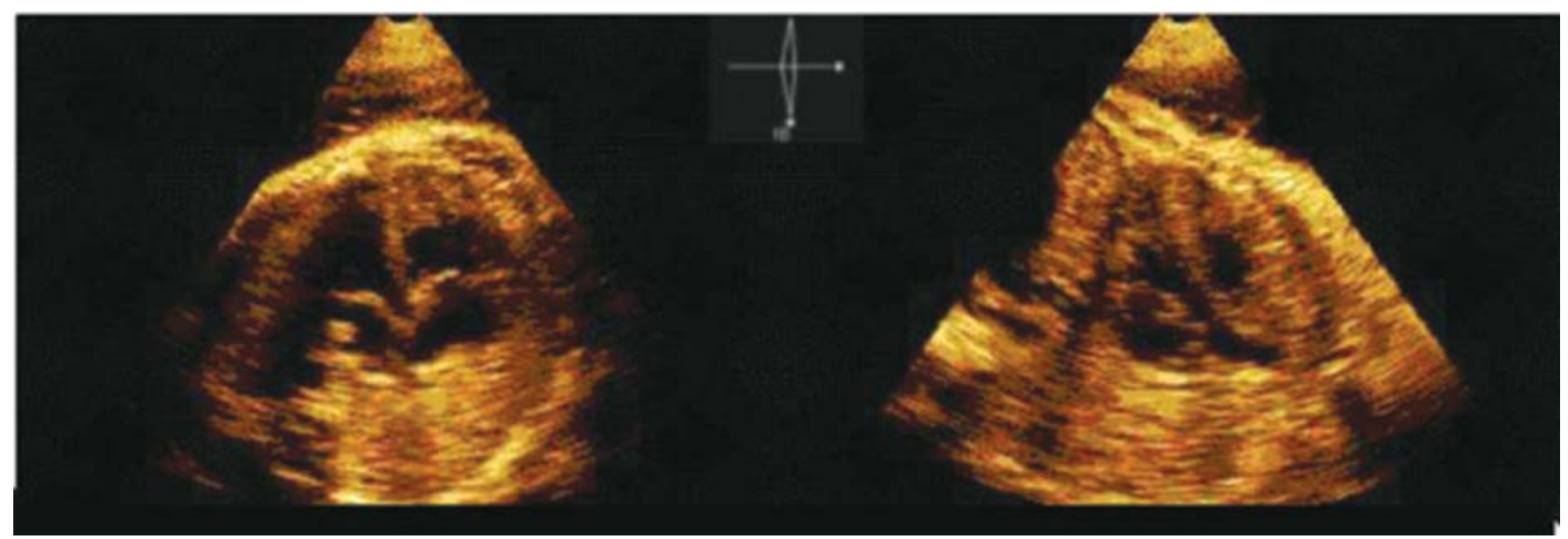

Fig. 6: Real-time simultaneous visualization of the axial plane with the four-chamber view (left-hand side of the figure), and of a perpendicular plane showing the left ventricular outflow tract (right-hand side). The reciprocal spatial orientation of the two planes is represented schematically between the two images

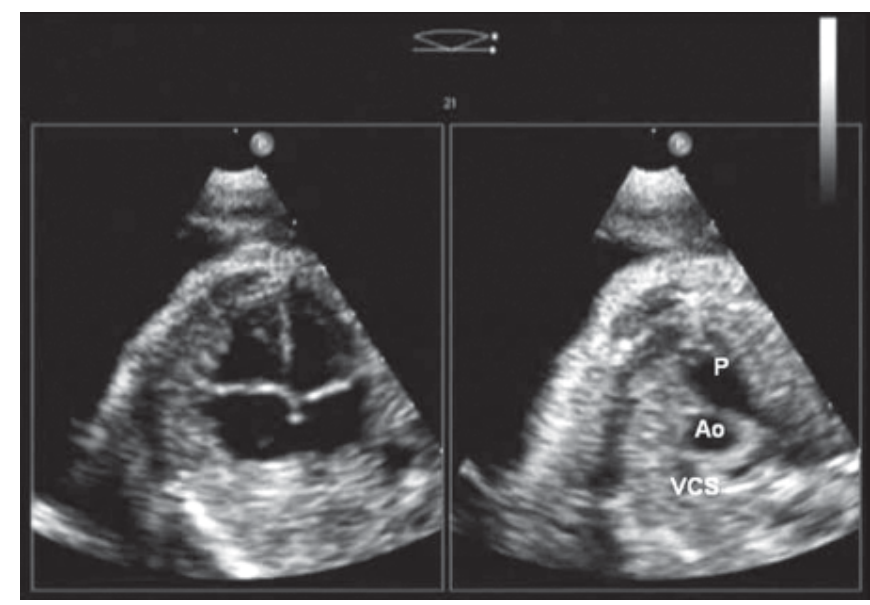

Fig. 7: When the second plane is oriented parallel and cranially to the axial plane of the four-chamber view (as shown by the scheme on the top of the Figure, between the two images), the 3 vessel view, with pulmonary artery $(\mathrm{P})$, aorta (Ao) and superior vena cava (VCS), can be simultaneously seen on the right-hand side of the screen frequency, reduced detail definition and impossibility to combine color Doppler with real-time 3D imaging. However, real-time 3D improves the overall understanding of anatomical structure arrangement. Therefore, real-time 3D ultrasound does not substitute but integrates conventional 2D and Doppler assessment of cardiovascular structures. Future improvements will allow to overcome the high costs and the limitations in image resolution in currently manufactured matrix array transducers.

\section{REFERENCES}

1. Simpson J, Weiner E, (Eds). Oxford English Dictionary. Oxford: Oxford University Press; 1989.

2. Sklansky MS, Nelson T, Strachan M, Pretorius D. Real-time three-dimensional fetal echocardiography: initial feasibility study. J Ultrasound Med 1999;18:745-52.

3. Scharf A, Geka F, Steinborn A, Frey H, Schlemmer A, Sohn C. 3D real-time imaging of the fetal heart. Fetal Diagn Ther 2000;15:267-74. 
4. Deng J, Sullivan ID, Yates R, Vogel M, McDonald D, Linney $\mathrm{AD}$, Rodeck $\mathrm{CH}$, Anderson RH. Real-time three-dimensional fetal echocardiography —optimal imaging windows. Ultrasound Med Biol 2002;28:1099-105.

5. Maulik D, Nanda NC, Singh V, Dod H, Vengala S, Sinha A, Sidhu MS, Khanna D, Lysikiewicz A, Sicuranza G, Modh N. Live three-dimensional echocardiography of the human fetus. Echocardiography 2003;20:715-21.

6. Sklansky MS, DeVore GR, Wong PC. Real-time 3-dimensional fetal echocardiography with an instantaneous volume-rendered display: early description and pictorial essay. J Ultrasound Med 2004;23:283-9.
7. Acar P, Dulac Y, Taktak A, Abadir S. Real-time threedimensional fetal echocardiography using matrix probe. Prenat Diagn 2005;25:370-5.

8. Sklansky M, Miller D, Devore G, Kung G, Pretorius D, Wong $\mathrm{P}$, Chang RK. Prenatal screening for congenital heart disease using real-time three-dimensional echocardiography and a novel 'sweep volume' acquisition technique. Ultrasound Obstet Gynecol 2005;25:435-43.

9. Goncalves LF, Espinoza J, Kusanovic JP, Lee W, Nien JK, Santolaya-Forgas J, Mari G, Treadwell MC, Romero R. Applications of 2-dimensional matrix array for 3- and 4dimensional examination of the fetus: a pictorial essay. J Ultrasound Med 2006;25:745-55. 